\title{
Vagus Nerve Stimulation in Refractory Epilepsy: Effects on Pro- and Anti-Inflammatory Cytokines in Peripheral Blood
}

\author{
H.J.M. Majoie ${ }^{a, b}$ K. Rijkers ${ }^{c}$ M.W. Berfelo ${ }^{c}$ J.A.R.J. Hulsman ${ }^{a} \quad$ A. Myint ${ }^{d}$ \\ M. Schwarz ${ }^{\text {d J.S.H. Vles }}{ }^{b}$ \\ ${ }^{a}$ Epilepsy Center Kempenhaeghe, Heeze, Departments of ${ }^{b}$ Neurology, and ${ }^{c}$ Neurosurgery, School of \\ Mental Health and Neuroscience, University Medical Centre Maastricht, Maastricht, The Netherlands; \\ dLaboratory for Psychoneuroimmunology and Therapeutic Drug Monitoring, University Psychiatric Hospital, \\ University of Munich, Munich, Germany
}

\section{Key Words}

Inflammation • Anticholinergic reflex · Tryptophan •

Serotonin $\cdot$ Kynurenin $\cdot$ Neurostimulation

\begin{abstract}
Objective: The vagus nerve has important immunological functions that may be relevant for its anticonvulsive action. We postulate that this anticonvulsive action is activated by a shift in the immune system resulting in a reduction of neurotoxic and an increase of neuroprotective tryptophan metabolites. Methods: Eleven patients with refractory epilepsy and 11 controls matched for age and gender were included in this study. The primary outcome measure was a $50 \%$ seizure reduction. Other variables were pro-inflammatory cytokines IL- 6 and TNF- $\alpha$, anti-inflammatory cytokine IL-10, cortisol, and the tryptophan metabolites 3-hydroxykynurenine (3-OH-KYN), kynurenic acid (KYNA), kynurenine, serotonin (5-HT) and 5-hydroxyindol acetic acid (5-HIAA). Blood samples were scheduled during baseline, and in week 28 of add-on treatment. Results: IL- 6 levels were higher in the responders than in the control group, and decreased after vagus nerve stimulation (VNS), whereas IL-10 was low and increased after VNS. In nonresponders, VNS resulted in an increase of IL-6 plasma levels and in a decrease of IL-10. Cor-
\end{abstract}

tisol concentrations are higher in the epilepsy group than in the control group. After VNS, these concentrations decreased. The concentrations of the tryptophan metabolites were lower in the epilepsy group than in the control group. The KYNA ratios are defined as the ratio of neuroprotective KYNA versus neurotoxic 3-OH-KYN and KYNA versus neurotoxic kynurenine: these ratios were lower in epilepsy patients than in controls, and they both moderately increased after VNS. Conclusion: The outcome of this preliminary study indicates that VNS causes a rebalancing of the immune system. This results in: (1) a reduction of neurotoxic and an increase of neuroprotective kynurenine metabolites and (2) in the normalization of cortisol levels.

Copyright $\odot 2010$ S. Karger AG, Basel

\section{Introduction}

Vagus nerve stimulation (VNS) is an alternative treatment for intractable epilepsy. The mode of action is assumed to be the result of modulation of deep brain structures by neuroactive substances. The severity of epileptic seizures is causally related to central inflammatory and neurotransmitter functions [1-5].

\section{KARGER}

Fax +4161306 1234 E-Mail karger@karger.ch www.karger.com
(C) 2010 S. Karger AG, Base

1021-7401/11/0181-0052\$38.00/0

Accessible online at:

www.karger.com/nim
H.J.M. Majoie, $\mathrm{MD}, \mathrm{PhD}$

Epilepsy Center Kempenhaeghe, PO Box 6

NL-5590 AB Heeze (The Netherlands)

Tel. +31 402279263 , Fax +31 402265691

E-Mail majoiem@kempenhaeghe.nl 


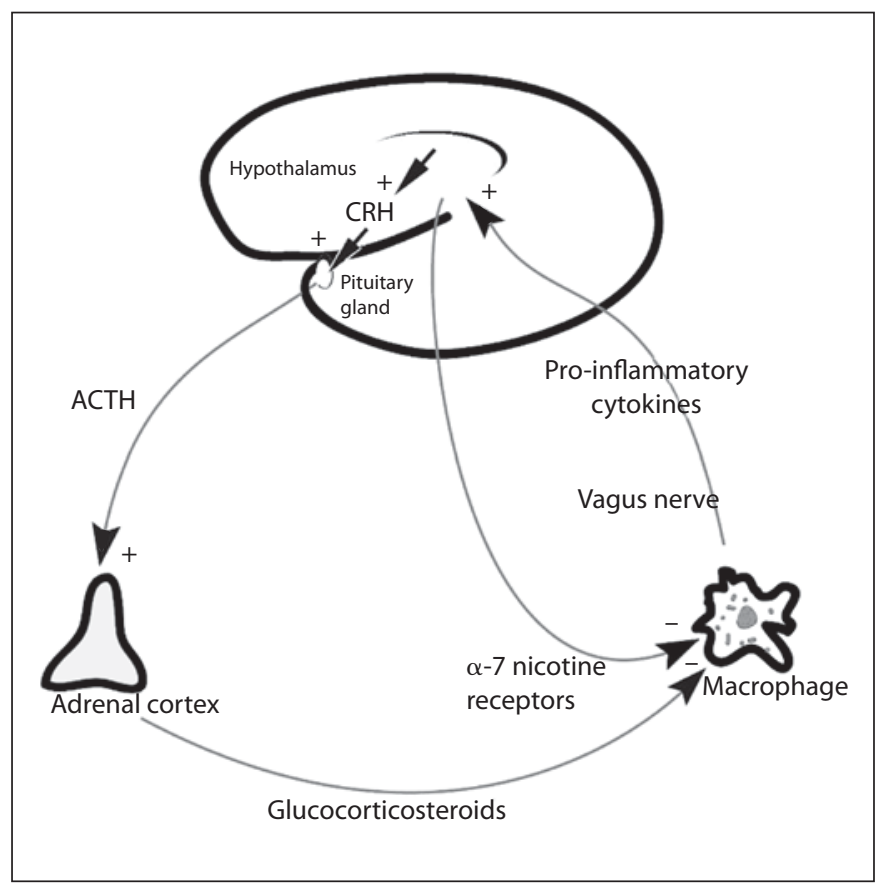

Fig. 1. The anticholinergic reflex loop.

Tryptophan metabolites are involved in the stimulation of glutaminergic N-methyl-D-aspartate (NMDA) receptors. Overstimulation of NMDA receptors has been implicated in the generation of seizures $[6,7]$. The vagus nerve has a major impact in attenuating inflammatory responses via the so-called anticholinergic reflex loop [8] (fig. 1). The pro-inflammatory cytokines influence the serotonin (5-HT) and kynurenine metabolism [7-9]. VNS also results in changes of tryptophan metabolite concentrations in the brain $[10,11]$ (fig. 2). However, the exact mechanism behind seizure frequency reduction and improvement of well-being in VNS is still unknown.

We postulate that the beneficial effect of VNS is activated by a shift in the immune system, resulting in a reduction of neurotoxic and an increase of neuroprotective tryptophan metabolites.

In the presented pilot study we aimed at: (1) evaluating the effectiveness of VNS, (2) evaluating the effects of VNS on the immune system and changes in the tryptophan metabolic pathway, and (3) linking the therapeutic effect of VNS to changes in the tryptophan metabolic pathway.

Neuroimmunomodulation in Epilepsy

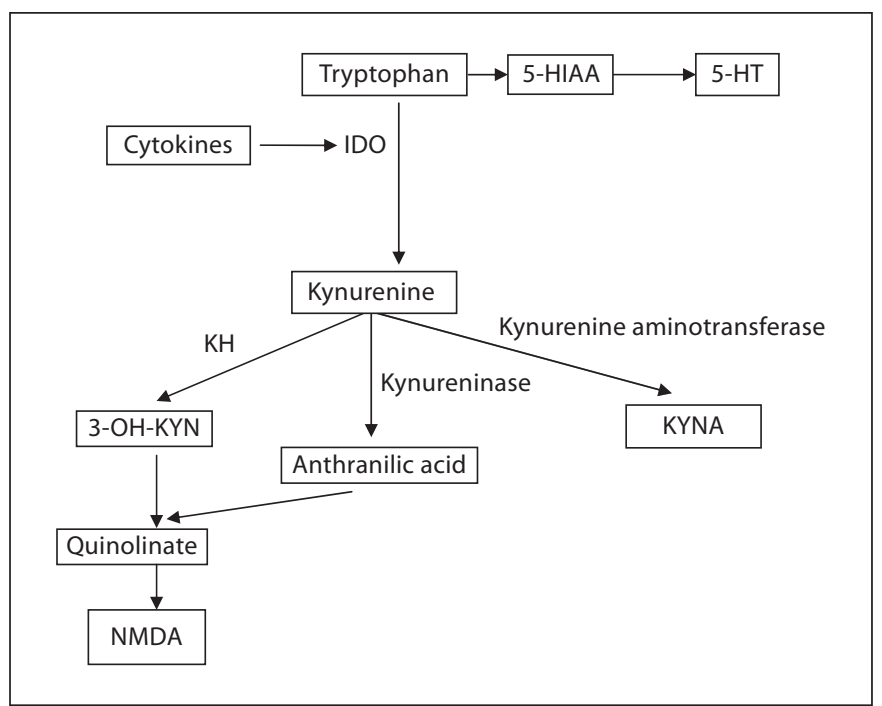

Fig. 2. $5-\mathrm{HT}=$ Serotonin; $3-\mathrm{OH}-\mathrm{KYN}=3$-hydroxykynurenine; KYNA = kynurenic acid; IDO = indoleamine $(2,3)$-dioxygenase; $\mathrm{KH}=$ kynurenine hydroxylase.

\section{Subjects and Methods}

We studied 11 patients with intractable epilepsy and 11 controls matched for age and gender. Patients and controls with known immune compromising diseases, infectious diseases or pregnancy were excluded. No changes in medication were allowed during the study. The study was approved by the medical ethics committee.

Seizure frequency per month at 6 months of VNS was compared with seizure frequency per month at baseline. The primary endpoint was a seizure frequency reduction of $50 \%$ or more. The main variables were: demographics, epilepsy-specific data, and biochemical and neuro-immunological variables in peripheral blood. Blood sampling was scheduled during baseline and in week 28 of add-on treatment. The time interval between the last seizure and blood sampling was $24 \mathrm{~h}$ or more.

IL-6 and TNF- $\alpha$ (pro-inflammatory cytokines), IL-10 (anti-inflammatory cytokine), 5-HT, kynurenine, kynurenic acid (KYNA), 3-hydroxykynurenine (3-OH-KYN), and 5-hydroxyindol acetic acid (5-HIAA) were assessed. The 'neuroprotective ratio' was determined to evaluate whether the effect of VNS is neuroprotective or neurotoxic. As we were unable to determine the concentration of quinolinic acid (neurotoxin), the KYNA/kynurenine and KYNA/3-OH-KYN ratios were determined to assess the balance between the neuroprotective and neurotoxic arm of the kynurenine metabolism. EDTA blood was collected and centrifuged. It was stored at $-70^{\circ} \mathrm{C}$ until analysis was performed. For determination, $7 \mathrm{~mm}$ of plasma were used.

The cytokine assessments were performed with an ELISA technique (R\&D Quantikine HS). In some samples, the cytokine concentrations were below the detection level. The level of detection of IL- 6 was $0.4 \mathrm{pg} / \mathrm{ml}, 1 \mathrm{pg} / \mathrm{ml}$ for TNF- $\alpha$ and $1.5 \mathrm{pg} / \mathrm{ml}$ for IL-10. While results below the level of detection cannot be regard- 
Table 1. Peripheral blood samples

\begin{tabular}{|c|c|c|c|c|c|c|c|c|}
\hline & Control & $\mathrm{n}$ & Baseline & $\mathrm{n}$ & After & $\mathrm{n}$ & $\mathrm{p}^{1}$ & $\mathrm{p}^{2}$ \\
\hline IL-6, pg/ml & $0.68(0.2-1.57)$ & 8 & $0.50(0.2-1.45)$ & 7 & $0.61(0.2-1.42)$ & 6 & 0.75 & 0.37 \\
\hline TNF- $\alpha, p g / m l$ & $0.5(0.5-1.89)$ & 4 & $1.03(0.5-2.39)$ & 6 & $0.5(0.5-2.56)$ & 2 & 0.65 & 0.74 \\
\hline IL-10, pg/ml & $1.68(0.75-34.12)$ & 7 & $2.45(0.75-21.59)$ & 8 & $2.13(0.75-15.18)$ & 10 & 0.9 & 0.15 \\
\hline Tryptophan, $\mu \mathrm{g} / \mathrm{ml}$ & $11.3(8-14.6)$ & 11 & $10.8(4.6-15.3)$ & 11 & $9.8(4.2-352.8)$ & 11 & 0.52 & 0.66 \\
\hline 5-HIAA, ng/ml & $7.8(2.7-41.5)$ & 11 & $3.6(2.6-9.5)$ & 11 & $6.0(2.3-7)$ & 11 & 0.08 & 0.29 \\
\hline KYNA, ng/ml & $6.6(5.1-14.9)$ & 11 & $4.8(3.7-17.6)$ & 9 & $6.4(3.3-10)$ & 8 & 0.24 & 0.75 \\
\hline 3-OH-KYN, ng/ml & $9.2(7.5-11.2)$ & 11 & $9.1(6.3-12)$ & 11 & $9.8(8.2-12.4)$ & 11 & 0.9 & 0.92 \\
\hline Kynurenine, ng/ml & $503.5(402.9-873.4)$ & 11 & $399.5(277-742.7)$ & 11 & $317.4(14.1-593)$ & 11 & 0.07 & 0.18 \\
\hline KYNA/3-OH-KYN & $0.72(0.52-1.99)$ & 11 & $0.64(0.36-1.71)$ & 9 & $0.65(0.5-2.67)$ & 8 & 0.4 & 0.92 \\
\hline KYNA $\cdot 1,000 /$ kynurenine & $13(7.1-37)$ & 11 & $12.8(5.79-47.86)$ & 9 & $16.1(11.92-156.13)$ & 8 & 0.9 & 0.46 \\
\hline
\end{tabular}

Values are medians (min.-max.). $\mathrm{n}=$ Number of samples.

${ }^{1}$ Mann-Whitney test (control - baseline). ${ }^{2}$ Wilcoxon (baseline - after VNS).

ed as missing, they cannot be regarded as true values. We therefore assumed IL- 6 values $<0.4 \mathrm{pg} / \mathrm{ml}$ to be $0.2 \mathrm{pg} / \mathrm{ml}$, TNF- $\alpha$ $<1 \mathrm{pg} / \mathrm{ml}$ to be $0.5 \mathrm{pg} / \mathrm{ml}$ and IL- $10<1.5 \mathrm{pg} / \mathrm{ml}$ to be $0.75 \mathrm{pg} / \mathrm{ml}$.

Tryptophan, 5-HT and kynurenine metabolites were assessed by use of HPLC.

Non-parametric statistics were used to analyze the data. The Mann-Whitney test was used to compare the controls with the baseline group. The Wilcoxon test was used to compare the results at baseline with the results after 28 weeks of treatment.

\section{Results}

The control group consisted of 6 males and 5 females with ages ranging from $10-64$ years (median age: 28 ). The epilepsy group also consisted of 6 males and 5 females. The ages in the epilepsy group ranged from $12-58$ years (median age: 30 ).

Six patients had cryptogenic localization-related epilepsy, four had symptomatic epilepsy and one had generalized epilepsy. The median duration of the epilepsy was 19 years. The patients had used 5-15 drugs before. One patient had been operated on and one had been on a ketogenic diet.

The reduction of the mean seizure frequency was $21 \%$. The responder rate was $36 \%$.

VNS resulted in an increase of the IL-6 (pro-inflammatory) plasma levels and in a decrease in IL-10 (antiinflammatory) plasma levels, thus converging to the values of the control group. TNF- $\alpha$ concentrations were above the detection level in only $33 \%$ of the samples, which makes it difficult to interpret the results. For IL-10 and IL-6, 76 and $64 \%$ of the measured concentrations were above the detection level.

Most of the responders had detectable cytokine levels. In this subgroup of responders, we measured a decrease in the pro-inflammatory cytokine IL- 6 in all responders. An increase of anti-inflammatory cytokine IL-10 after VNS was seen in two out of four responders. The responders who already showed high levels of IL-10 $(n=2)$ did not show a further increase after VNS. None of the differences reached a level of statistical significance.

Epilepsy patients had a higher cortisol concentration than controls (346 vs. $326 \mathrm{nmol} / \mathrm{l}$ ). After VNS, the levels decreased $(286 \mathrm{nmol} / \mathrm{l})$.

In the subgroup of responders, three out of four patients showed a decrease of cortisol concentrations after VNS. The one patient whose cortisol level did not change after VNS already had a low cortisol level at baseline.

Most tryptophan metabolites were lower in epilepsy patients than in healthy individuals. After treatment, kynurenine and tryptophan decreased. We noticed an increase in the serotonin metabolite 5-HIAA and kynurenine metabolites KYNA and 3-OH-KYN. None of the differences reached a level of statistical significance (table 1). Only the KYNA assessment had missing values (2 samples at baseline and 3 samples after VNS).

Epilepsy patients showed lower KYNA ratios than controls. These ratios moderately increased after VNS (table 1). Unfortunately, three of five missing values occurred in the subgroup of responders $(n=4)$, leaving us with only one responder in whom we were able to assess the baseline and post-VNS KYNA/3-OH-KYN ratio. In 
this patient, the neuroprotective ratio was increased after VNS because the increase in KYNA was larger than the increase in 3-OH-KYN.

\section{Discussion}

A responder rate of $36 \%$ is in line with the results known from observational and controlled studies on VNS in epilepsy $[12,13]$.

Based on the literature, one would expect a pro-inflammatory status in patients with epilepsy $[3,14,15]$. Our preliminary data did not confirm the previous findings. Again, based on the literature one would expect VNS to provoke an anti-inflammatory reaction $[3,4,16-$ 19]. Our preliminary data did not confirm this observation either. An increased pro-inflammatory reaction after VNS was also described by Corcoran et al. [20], who studied cytokine reactions in 10 patients with therapyresistant depression. Nevertheless, marked increases in pro-inflammatory cytokines are presumably non-therapeutic. Our findings are in line with our expectations only if we analyze the responders as a subgroup: most responders showed a pro-inflammatory status before VNS treatment and an anti-inflammatory reaction after VNS. Those responders who already had high anti-inflammatory IL-10 levels at baseline did not show a further increase after VNS.

The decrease of 5-HIAA concentration in intractable epilepsy patients that we observed can be explained by the following hypothesis, which is also explained in figure 2. According to this neurodegeneration hypothesis, increased levels of pro-inflammatory cytokines influence the tryptophan metabolism, resulting in decreased metabolism of tryptophan to 5-HT and increased me- tabolism of tryptophan to kynurenine. This subsequently leads to an imbalance between the neuroprotective NMDA receptor antagonist KYNA and the neurotoxic 3-OH-KYN [7-9]. The reduced KYNA/kynurenine and $\mathrm{KYNA} / 3-\mathrm{OH}-\mathrm{KYN}$ ratios in epilepsy patients are in line with this neurodegeneration hypothesis. These results are indicative of an imbalance between neuroprotective and neurotoxic metabolites in epilepsy patients, and with previous reports showing significantly lower levels of neuroprotective KYNA in children with intractable epilepsy than in healthy controls [21-23].

Hypothalamic pituitary adrenal axis dysfunction or a state of hypercortisolemia can be correlated with cognitive deficits specific to medial temporal lobe memory, depression and epilepsy $[24,25]$. The high cortisol levels at baseline and the decrease after VNS are in line with these studies and with our anti-inflammatory concept.

The purpose of this preliminary study was to determine which parameters were of value in assessing the anti-inflammatory action of VNS as a possible explanation of its therapeutic action. The interpretation of our results is only tentative due to the small number of patients, the rapid changes in cytokine concentrations, and the involvement of several central and peripheral networks. However, it appears that epilepsy patients who respond to VNS show a trend towards rebalancing the immune system as well as the 5-HT and kynurenine metabolism.

\section{Acknowledgements}

The authors are grateful to Ton Broekman and Anouk Bol for the immune assessments and to Johanna Zach for analyzing the metabolites of the serotonin and kynurenic pathway. K.R. is supported by grant No. 92003453 from the Dutch Scientific Organisation (ZonMW).

\section{References}

1 Salgado-Commissariat D, Alkadhi KA: Serotonin inhibits epileptiform discharge by activation of 5-HT1A receptors in CA1 pyramidal neurons. Neuropharmacology 1997; 36:1705-1712.

2 Shouse MN, Staba RJ, Saquib SF, Farber PR: Long-lasting effects of feline amygdala kindling on monoamines, seizures and sleep. Brain Res 2001;892:147-165.

3 Hulkkonen J, Koskikallio E, Rainesalo S, Keranen T, Hurme M, Peltola J: The balance of inhibitory and excitatory cytokines is differently regulated in vivo and in vitro among therapy resistant epilepsy patients. Epilepsy Res 2004;59:199-205.
4 Vezzani A, Moneta D, Richichi C, Aliprandi M, Burrows SJ, Ravizza T, Perego C, De Simoni MG: Functional role of inflammatory cytokines and antiinflammatory molecules in seizures and epileptogenesis. Epilepsia 2002;43(suppl 5):30-35.

5 Chugani DC, Chugani HT: Does serotonin have trophic effects in temporal lobe epilepsy? Neurology 2003;60:736-737.

6 Heyes MP, Saito K, Crowley JS, Davis LE, Demitrack MA, Der M, Dilling LA, Elia J, Kruesi MJ, Lackner A, et al: Quinolinic acid and kynurenine pathway metabolism in inflammatory and non-inflammatory neurological disease. Brain 1992;115:1249-1273.
7 Heyes MP, Wyler AR, Devinsky O, Yergey JA, Markey SP, Nadi NS: Quinolinic acid concentrations in brain and cerebrospinal fluid of patients with intractable complex partial seizures. Epilepsia 1990;31:172-177.

8 Gallowitsch-Puerta M, Tracey KJ: Immunologic role of the cholinergic anti-inflammatory pathway and the nicotinic acetylcholine alpha 7 receptor. Ann NY Acad Sci 2005; 1062:209-219.

9 Myint AM, Kim YK: Cytokine-serotonin interaction through IDO: a neurodegeneration hypothesis of depression. Med Hypotheses 2003;61:519-525. 
10 Ben Menachem E, Hamberger A, Hedner T, Hammond EJ, Uthman BM, Slater J, Treig T, Stefan H, Ramsay RE, Wernicke JF, et al: Effects of vagus nerve stimulation on amino acids and other metabolites in the CSF of patients with partial seizures. Epilepsy Res 1995;20:221-227.

11 Hammond EJ, Uthman BM, Wilder BJ, Ben Menachem E, Hamberger A, Hedner T, Ekman R: Neurochemical effects of vagus nerve stimulation in humans. Brain research 1992;583:300-303.

12 Cramer JA, Ben Menachem E, French J: Review of treatment options for refractory epilepsy: new medications and vagal nerve stimulation. Epilepsy Res 2001;47:17-25.

13 Majoie HJ, Berfelo MW, Aldenkamp AP, Renier WO, Kessels AG: Vagus nerve stimulation in patients with catastrophic childhood epilepsy, a 2-year follow-up study. Seizure 2005;14:10-18.

14 Vezzani A, Balosso S, Ravizza T: The role of cytokines in the pathophysiology of epilepsy. Brain Behav Immun 2008;22:797-803.
15 Liimatainen S, Fallah M, Kharazmi E, Peltola M, Peltola J: Interleukin-6 levels are increased in temporal lobe epilepsy but not in extra-temporal lobe epilepsy. J Neurol 2009; 256:796-802.

16 Hosoi T, Okuma Y, Nomura Y: The mechanisms of immune-to-brain communication in inflammation as a drug target. Curr Drug Targets Inflamm Allergy 2002;1:257-262.

17 Pavlov VA, Tracey KJ: The cholinergic antiinflammatory pathway. Brain Behav Immun 2005; 19:493-499.

18 Tracey KJ: The inflammatory reflex. Nature 2002;420:853-859.

19 De Herdt V, Bogaert S, Bracke KR, Raedt R, De Vos M, Vonck K, Boon P: Effects of vagus nerve stimulation on pro- and anti-inflammatory cytokine induction in patients with refractory epilepsy. J Neuroimmunol 2009; 214:104-108.

20 Corcoran C, Connor TJ, O’Keane V, Garland MR: The effects of vagus nerve stimulation on pro- and anti-inflammatory cytokines in humans: a preliminary report. Neuroimmunomodulation 2005;12:307-309.
21 Yamamoto H, Shindo I, Egawa B, Horiguchi $\mathrm{K}$ : Kynurenic acid is decreased in cerebrospinal fluid of patients with infantile spasms. Pediatr Neurol 1994;10:9-12.

22 Yamamoto H: Studies on CSF tryptophan metabolism in infantile spasms. Pediatr Neurol 1991;7:411-414.

23 Giroud M, Dumas R, Dauvergne M, D'Athis P, Rochette L, Beley A, Bralet J: 5-Hydroxyindoleacetic acid and homovanillic acid in cerebrospinal fluid of children with febrile convulsions. Epilepsia 1990;31:178-181.

24 Jameison K, Dinan TG: Glucocorticoids and cognitive function: from physiology to pathophysiology. Hum Psychopharmacol 2001;16:293-302

25 Galimberti CA, Magri F, Copello F, Arbasino C, Cravello L, Casu M, Patrone V, Murialdo G: Seizure frequency and cortisol and dehydroepiandrosterone sulfate (DHEAS) levels in women with epilepsy receiving antiepileptic drug treatment. Epilepsia 2005; 46:517-523. 